\title{
Formation of a supramolecular chromophore: a spectroscopic and theoretical study $\dagger$
}

\author{
Andreas Bernet, ${ }^{a b}$ Rodrigo Q. Albuquerque, ${ }^{* b c}$ Marina Behr, ${ }^{a b}$ Sebastian T. Hoffmann ${ }^{d}$ \\ and Hans-Werner Schmidt*ab
}

Received 20th September 2011, Accepted 8th November 2011

DOI: $10.1039 / \mathrm{c} 1 \mathrm{sm} 06789 \mathrm{c}$

\begin{abstract}
Spectroscopic and theoretical investigations of the self-assembly of a particular 1,3,5-benzene trisamide-based low molecular weight hydrogelator are described. This trisamide is $\mathrm{pH}$-sensitive, and surprisingly forms a photoluminescent supramolecular hydrogel. Controlled gel formation in combination with the luminescence properties allows studying the self-assembly process in detail. The experimental results are confirmed by Density Functional Theory (DFT) calculations, revealing that the photoluminescence originates from the formation of a supramolecular chromophore.
\end{abstract}

The interest in multichromophoric assemblies has grown in the last few years, due to their potential applications as advanced functional materials. ${ }^{1}$ A fascinating bottom-up approach to realize supramolecular multichromophore systems is based on the self-assembly of low molecular weight organic compounds. Fine-tuning of the underlying molecular structure allows for a guided self-assembly into hierarchical superstructures. One particularly interesting structural motif of a supramolecular self-assembly system is based on benzene 1,3,5-tricarboxamide (BTA) derivatives. ${ }^{2}$ BTA-based molecules selfassemble into supramolecular columns in solution and in solid state ${ }^{3}$ and form liquid crystalline phases and well-defined nanostructures. ${ }^{4}$ This enables applications as thickening and gelation agents, ${ }^{5}$ nucleation and clarifying agents ${ }^{6}$ and electret additives ${ }^{7}$ for thermoplastic polymers.

Here, we present the self-assembly of the polar BTA derivative 1 (Fig. 1a) in aqueous media. We found that $\mathbf{1}$ featuring three $p$-carboxylphenyl side arms is one of the very rare examples ${ }^{8}$ of a BTA derivative that is able to form supramolecular hydrogels. The resulting gels are fully $\mathrm{pH}$-reversible, but thermostable up to $100{ }^{\circ} \mathrm{C}$

${ }^{a}$ Macromolecular Chemistry I, University of Bayreuth, Bayreuth, 95440, Germany. E-mail: hans-werner.schmidt@uni-bayreuth.de; Fax: +49921 553206; Tel: +49921553200

${ }^{b}$ Bayreuth Institute for Macromolecular Research (BIMF), University of Bayreuth, Bayreuth, 95440, Germany

'Theoretical Physics IV, University of Bayreuth, Bayreuth, 95440, Germany. E-mail: rqa_ufpe@yahoo.com; Fax: +49 92155 3223; Tel: +49921553363

${ }^{d}$ Experimental Physics II, University of Bayreuth, Bayreuth, 95440, Germany

$\dagger$ Electronic supplementary information (ESI) available: Experimental and computational details of all optimized structures. See DOI: $10.1039 / \mathrm{c} 1 \mathrm{sm} 06789 \mathrm{c}$ even at the critical gelation concentration of $2 \mathrm{~g} \cdot \mathrm{L}^{-1}$. To the best of our knowledge, this is the only thermostable example of a supramolecular $\mathrm{pH}$-reversible hydrogel system. The outstanding properties of the hydrogels will be reported in detail elsewhere.

We also discovered that the assemblies of $\mathbf{1}$ present in the bulk and in the gel state exhibit a strong blue emission upon UV irradiation (Fig. 1b). Although 1 has been reported to be used for the production of branched polyamides ${ }^{9}$ and metal-organic frameworks, ${ }^{10}$ we were the first to discover its ability to form supramolecular hydrogels and their unexpected photoluminescence (PL) properties. In the literature, a single BTA-derived organogelator that features photoluminescence in the gel state in aprotic organic solvents is reported. ${ }^{11}$ It was concluded that the PL activity arises from molecular aggregation via H-bonding, but no further detailed explanation of the origin of the PL was given.

Herein, we investigate the self-assembly of $\mathbf{1}$ leading to a photoluminescent hydrogel. Featuring three carboxy moieties, the aggregation behavior of $\mathbf{1}$ in aqueous environment is controllable by a)
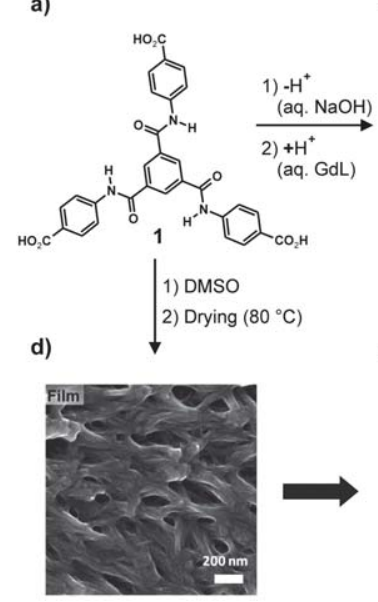

b)

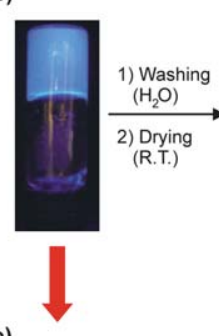

c)

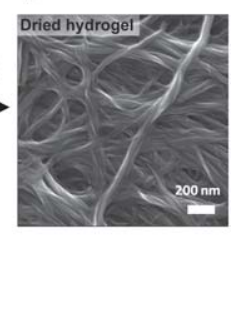

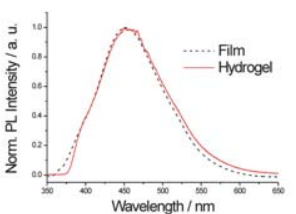

Fig. 1 (a) Chemical structure of the investigated 1,3,5-benzene trisamide 1 (GdL: glucono-delta-lactone). (b) Photograph of a macroscopic hydrogel sample of $10 \mathrm{~g} \cdot \mathrm{L}^{-1}$ of 1 under UV irradiation $\left(\lambda_{\mathrm{exc}}=366 \mathrm{~nm}\right)$. (c) SEM micrograph of the supramolecular aggregates of $\mathbf{1}$ from dried hydrogel and (d) as obtained by drop-casting from DMSO solution. (e) PL spectra of 1 in the film and in the hydrogel state $\left(\lambda_{\mathrm{exc}}=330 \mathrm{~nm}\right)$. The corresponding absorption spectra are shown in the ESI (Fig. S2 $\dagger$ ). 
changing the $\mathrm{pH}$. When $\mathbf{1}$ is mixed with aqueous $\mathrm{NaOH}$ solution, it is transferred into the corresponding sodium salt $\mathbf{1 N a}$ which is readily water-soluble. Upon acidification the carboxylates of $\mathbf{1 N a}$ get protonated. This reduces the solubility, enables self-assembly and finally leads to the formation of a macroscopic hydrogel (Fig. 1b). Fig. 1c shows a SEM image of the dried hydrogel exhibiting a fibrillar three-dimensional network morphology. A different, but also nanofibrillar morphology is formed by casting a solution of $\mathbf{1}$ in DMSO and subsequent drying (Fig. 1d).

The steady-state PL spectra of $\mathbf{1}$ as dried film and in the hydrogel are shown in Fig. 1e. Although the morphologies obtained from the dried hydrogel and from the film sample look different, the obtained emission spectra are very similar. This indicates that the respective emitting excited states are the same, and that the morphological difference is only caused by different hierarchical superstructures of one common underlying assembly motif, caused by different conditions under which self-assembly of $\mathbf{1}$ takes place. Indeed, XRD spectra of both specimens (see ESI, Fig. S4 $\dagger$ ) indicate that in the film sample there is a much higher long-range order than in the xerogel sample. It has to be noted that PL was only observed in the presence of the supramolecular structures. In DMSO solutions at concentrations up to $10 \mathrm{~g} \cdot \mathrm{L}^{-1}$ of $\mathbf{1}$, no PL was detectable with a standard PL setup.

In order to investigate the formation of the luminescent supramolecular hydrogel, it is necessary to decrease the $\mathrm{pH}$ slowly in a continuous and controlled manner. This can be accomplished by mixing an aqueous solution of $\mathbf{1 N a}$ with glucono-delta-lactone (GdL). ${ }^{12}$ The hydrogel of $\mathbf{1}$ is then gradually formed, which enables recording the luminescence spectra over time (Fig. 2). ${ }^{13}$ Before the addition of GdL, no PL is observed (a). $2.5 \mathrm{~min}$ after addition, a first peak at ca. $380 \mathrm{~nm}$ is visible (b). This peak can be retrieved throughout the following absorption spectra (c to g). At $7.5 \mathrm{~min}$, a second peak evolves at $c a$. $450 \mathrm{~nm}$ and is increasing over time showing a slight red-shift (c to g).

Before the addition of GdL, the highly charged $\mathbf{1 N a}$ is molecularly dissolved due to electrostatic repulsion as indicated by the absence of significant scattering signal in DLS experiments. After the addition of GdL, protons are released by the hydrolysis of GdL. From a statistical point of view, it is reasonable to assume that at first only one carboxylate per molecule of $\mathbf{1 N a}$ gets protonated. As this lowers the solubility, the partially protonated $\mathbf{1 N a}$ molecules start aggregating upon encountering each other. Two aggregation modes are possible: dimerization of two molecules via the carboxylic groups leads to "side-by-side" dimers (ESI, Fig. S7 $†$ ), whereas the stacking of two molecules driven by triple hydrogen bonding of the amide groups and $\pi-\pi$ interaction of the aromatic cores yields energetically more favorable "on-top" dimers (ESI, Fig. S8 $†$ ). Over time, with the further decrease of $\mathrm{pH}$ more and more of the initially formed dimers can aggregate into more extended columnar superstructures.

So far, only a few computational studies on BTA derivatives have been reported, ${ }^{2,14}$ and the majority of the mentioned studies is focusing on the behavior of BTAs in organic solvents and polymer melts. Aiming to understand how the self-assembly of 1 influences the optical properties of the resulting aggregates, DFT and time-dependent DFT (TDDFT) calculations were carried out for the monomer, dimer, trimer and tetramer forms of $1 .^{15}$ The optimized geometries of monomer and tetramer (for dimers and trimer see ESI, Fig. S7-S9†) together with the highest occupied molecular orbital (HOMO) and lowest unoccupied molecular orbital (LUMO) are shown in Fig. 3a. a)

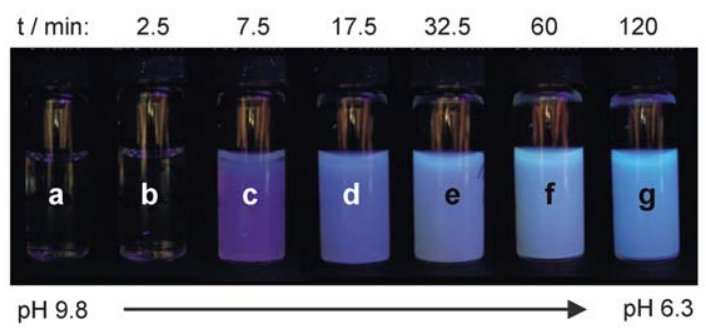

Formation of the supramolecular chromophores b)

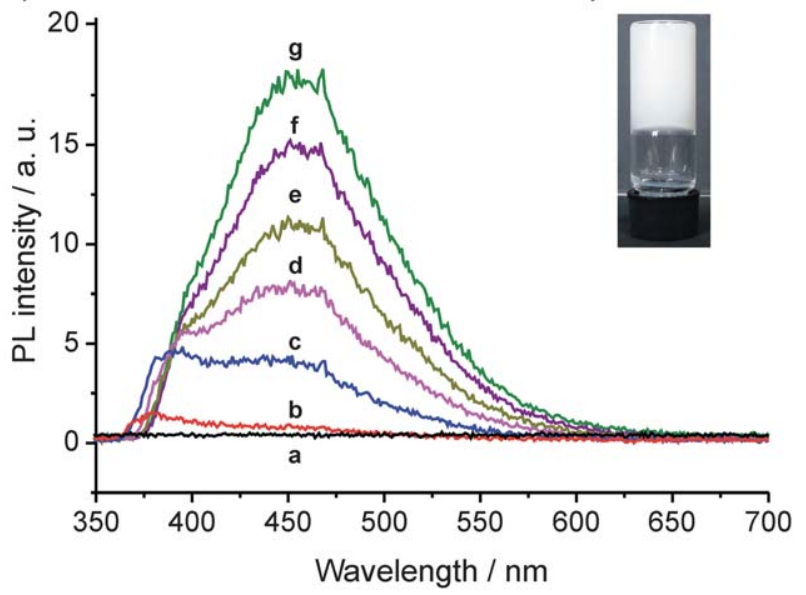

Fig. 2 (a) Photographs of a macroscopic sample of an aqueous solution of $10 \mathrm{~g} \cdot \mathrm{L}^{-1}$ of $\mathbf{1 N a}$ before (a) and after ( $\mathrm{b}$ to $\mathrm{g}$ ) addition of GdL (images were recorded under UV irradiation, $\lambda_{\text {exc }}=366 \mathrm{~nm}$. Differences in color are caused by scattering effects and automatic white balance of the camera). (b) Corresponding PL spectra $\left(\lambda_{\mathrm{exc}}=300 \mathrm{~nm}\right.$. The noise is caused by the fast scanning speed that was chosen in order to avoid significant changes of the spectra during acquisition). (c) Photograph of inverted hydrogel sample after $24 \mathrm{~h}$ (although the $\mathrm{pH}$ value continuously decreases reaching a value of $c a .4 .2$ after 4 days, there are no significant changes in the PL spectra compared to the sample after $2 \mathrm{~h}(\mathrm{~g})$ ).

In the case of dimer, trimer, and tetramer, columnar stacking of monomer units takes place due to $\pi-\pi$ interaction and triple hydrogen bonding of the benzene trisamide core. The presence of hydrogen-bonded amide units in dried hydrogel and bulk samples of 1 was experimentally proven by FT-IR (ESI, Fig. S5†). The calculated core distances are $3.37 \AA$ both for the dimer and trimer and $3.28 \AA$ for the tetramer, respectively. Although the dispersion correction seems to overestimate the $\pi-\pi$ interaction, the calculated values are still in reasonable agreement with the results of the X-ray analysis of a dried hydrogel sample (3.47 A, see ESI, Fig. S4 $\dagger$ ) and in a typical range for $\pi-\pi$ interacting systems. In addition to the interactions of the aromatic cores, $\pi-\pi$ interactions of the phenylene units of the side arms can be recognized, leading to a bending of the aromatic side arms. However, it is reasonable to assume that this edge effect will be evened out when increasing the number of monomer units in a supramolecular column. Additionally, hydrogen bonding between carboxylic groups of different columns is likely to occur. This also will counteract the bending of the side arms. Collectively, the computed noncovalent intermolecular interactions lead to a columnar stacking of monomers of 1 with a twist angle of $c a .60^{\circ}$. This fits very well in the packing model of BTA derivatives proposed by Meijer and co-workers. ${ }^{2}$ 
a)

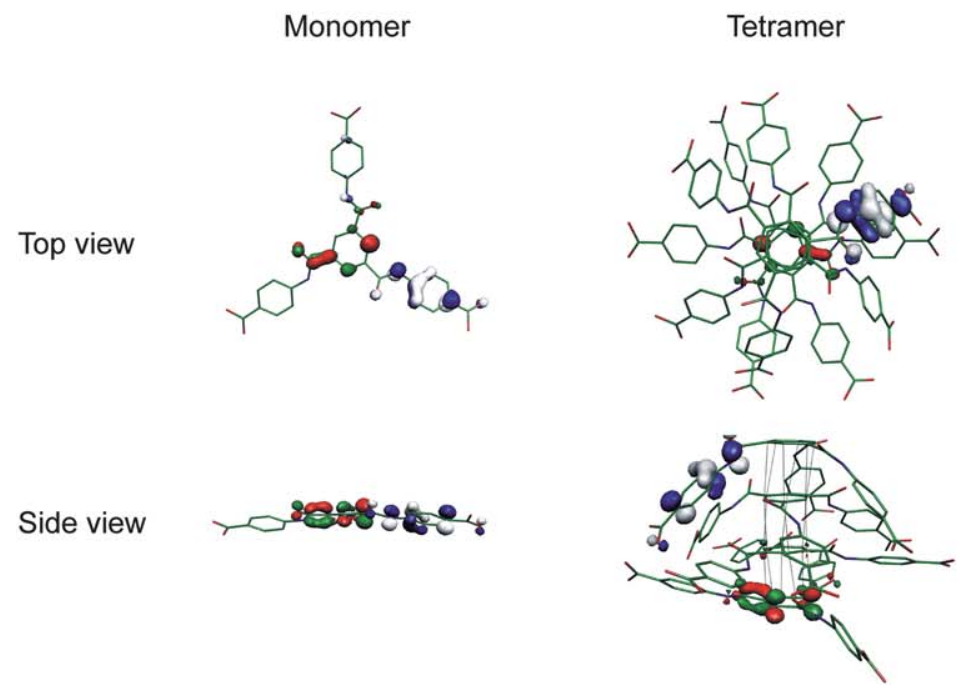

b)

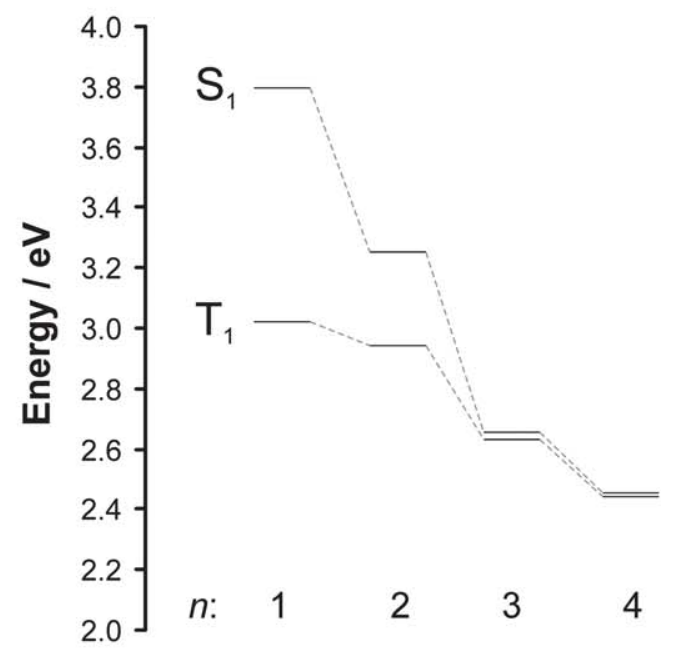

Fig. 3 (a) DFT optimized geometries of $\mathbf{1}$ as a monomer and a tetramer (top view and side view are shown) with the respective frontier molecular orbitals HOMO (blue/white) and LUMO (red/green). The atom labels are: carbon (green), nitrogen (blue), and oxygen (red). The hydrogens are omitted for clarity. The black dotted lines represent the stacking of the aromatic cores. (b) TDDFT calculated energies of the excited singlet $\left(\mathrm{S}_{1}\right)$ and triplet $\left(\mathrm{T}_{1}\right)$ states of aggregates of $\mathbf{1}$.The $n$ values refer to the number of monomer units of $\mathbf{1}$.

The calculated $S_{1}$ and $T_{1}$ excited states of the monomer and the "on-top" dimer, trimer and tetramer are basically localized on the central aromatic cores, which are less exposed to solvent molecules, and shielding effect becomes more pronounced upon increasing the number $n$ of monomer units of $\mathbf{1}$. This may explain the very similar emission spectra observed for supramolecular aggregates of $\mathbf{1}$ in different chemical environments as in the hydrogel and in the thin film. However, it has to be noted that the aggregates in the film and in the hydrogel are hierarchical superstructures of $\mathbf{1}$ with much larger $n$ values than the calculated aggregates. In Fig. $3 b$, the calculated energy levels for aggregates of $\mathbf{1}$ having different $n$ values are shown.

From monomer to tetramer, the energy levels are lowered, and the singlet-triplet splitting decreases. The strong hydrogen bonds between complementary amide groups inside the same supramolecular column, as well as $\pi-\pi$ interactions, cause a considerable red shift in the energies of those excited states. This reflects the experimental findings during the controlled gelation/PL experiments. We assign the first visible peak in the PL spectrum to the formation of an "on-top" dimer. The second detected peak and its red-shift over time fit quite well to the build-up of aggregates of $\mathbf{1}$ with an increasing $n$.

A "side-by-side" dimer of $\mathbf{1}$ as the origin of the second peak is unlikely, as TDDFT calculations show that the corresponding energy values of $S_{1}$ and $T_{1}$ are comparable to those found for the monomer (see ESI, Table S1 $\uparrow$ ), meaning that the experimentally observed red shift can only be reproduced by the calculations assuming the formation of the "on-top" dimer. Furthermore, we can also conclude that a bundling of several columns caused by dimerization of peripheral carboxylic groups of different columns has a negligible influence on the energy values of the underlying structures. This type of peripheral interactions leads to a rigidification of the whole supramolecular structure, thus resulting in smaller non-radiative rates and causing the well-known aggregation-induced emission enhancement. ${ }^{16}$
Additionally, DFT reveals that $S_{1}$ and $T_{1}$ computed for all aggregation forms of $\mathbf{1}$ are mostly described by HOMO $\rightarrow$ LUMO excitations. These states have a strong charge transfer (CT) character, the charge being transferred from the peripheral side groups to the central aromatic cores. ${ }^{17}$ Although HOMO and LUMO show only poor overlap, transitions to higher-lying excited states, which are described by $(\mathrm{HOMO}-x) \rightarrow(\mathrm{LUMO}+y)$ excitations, do possess much larger oscillator strength and still exhibit CT character. After absorption, energy transfer to defects may also play a role in the emission. ${ }^{18}$

In agreement with the literature, all amide groups within a supramolecular column are pointing to the same direction, giving rise to a strong macrodipole. ${ }^{19}$ This may explain the CT character of the excited states of supramolecular aggregates of $\mathbf{1}$, as a spatial charge separation can stabilize the macrodipole. However, it is important to remember that the structure shown in Fig. 3 is a very simplified model that helps to understand the experimental results. Therefore molecular dynamics simulations are necessary to achieve a more complete representation of the aggregates, since a great number of interactions are expected to be present in the film and in the gel.

\section{Conclusions}

The solid-state luminescence of $\mathbf{1}$ in combination with the $\mathrm{pH}$ controllable self-aggregation in aqueous media has proved to be highly useful to investigate the self-assembly process of the widely used BTA motif. DFT and experimental results strongly suggest that the blue luminescence recorded for the film and the hydrogel of $\mathbf{1}$ arises exclusively from supramolecular columnar stacks with a large number of monomers. The strong CT character of the calculated excited states, together with their well-shielded electron densities, suggest that $\mathbf{1}$ is an excellent candidate to be used as a model system for new functional supramolecular materials like non-covalent molecular wires. 


\section{Acknowledgements}

Financial support by the Deutsche Forschungsgemeinschaft (DFG) in the frame of Research Training Group GRK 1640 and Priority Program SPP 1259 is gratefully acknowledged. Synthesis of compound 1 was carried out within the Collaborative Research Centre SFB 840 (project B4). The German-Israeli Foundation is acknowledged for financial support. We thank B. Brunner for performing the elemental analysis (Prof. A. Jess, Department of Chemical Engineering, University of Bayreuth) and Martina Heider for the SEM experiments (Bayreuth Institute for Macromolecular Research, University of Bayreuth). We gratefully acknowledge Prof. Anna Köhler (Experimental Physics II, University of Bayreuth) for fruitful discussions.

\section{Notes and references}

1 E. Collini and G. D. Scholes, Science, 2009, 323, 369; B. Bodenant, F. Fages and M. H. Delville, J. Am. Chem. Soc., 1998, 120, 7511; O. Mirzov, R. Bloem, P. R. Hania, D. Thomsson, H. Z. Lin and I. G. Scheblykin, Small, 2009, 5, 1877; W. T. Wiesler, J. T. Vazquez and K. Nakanishi, J. Am. Chem. Soc., 1987, 109, 5586; J. Hofkens, M. Maus, T. Gensch, T. Vosch, M. Cotlet, F. Kohn, A. Herrmann, K. Mullen and F. De Schryver, J. Am. Chem. Soc., 2000, 122, 9278; J. Hofkens, M. Cotlet, T. Vosch, P. Tinnefeld, K. D. Weston, C. Ego, A. Grimsdale, K. Mullen, D. Beljonne, J. L. Bredas, S. Jordens, G. Schweitzer, M. Sauer and F. De Schryver, Proc. Natl. Acad. Sci. U. S. A., 2003, 100, 13146; J. W. Verhoeven, J. Photochem. Photobiol., C, 2006, 7, 40.

2 M. P. Lightfoot, F. S. Mair, R. G. Pritchard and J. E. Warren, Chem. Commun., 1999, 19, 1945; P. J. M. Stals, M. M. J. Smulders, R. Martín-Rapún, A. R. A. Palmans and E. W. Meijer, Chem.-Eur. J., 2009, 15, 2071.

3 P. J. M. Stals, J. C. Everts, R. de Bruijn, I. A. W. Filot, M. M. J. Smulders, R. Martín-Rapún, E. A. Pidko, T. F. A. de Greef, A. R. A. Palmans and E. W. Meijer, Chem.-Eur. J., 2010, 16, 810; T.-Q. Nguyen, R. Martel, P. Avouris, M. L. Bushey, L. Brus and C. Nuckolls, J. Am. Chem. Soc., 2004, 126, 5234.

4 C. Xue, F. Ilhan, S. Jin, S. Z. D. Cheng, M. A. Meador and R. K. Eby, Polym. Prepr. (Am. Chem. Soc., Div. Polym. Chem.), 2004, 45, 820; P. J. M. Stals, J. F. Haveman, R. Martín-Rapún, C. F. C. Fitié, A. R. A. Palmans and E. W. Meijer, J. Mater. Chem., 2009, 19, 124; Y. Matsunaga, N. Miyajima, Y. Nakayasu, S. Sakai and M. Yonenaga, Bull. Chem. Soc. Jpn., 1988, 61, 207.

5 Y. Yasuda, E. Iishi, H. Inada and Y. Shirota, Chem. Lett., 1996, 25, 575; K. Hanabusa, C. Koto, M. Kimura, H. Shirai and A. Kakehi, Chem. Lett., 1997, 26, 429; S. J. Lee, C. R. Park and J. Y. Chang, Langmuir, 2004, 20, 9513; M. de Loos, J. H. van Esch, R. M. Kellogg and B. L. Feringa, Tetrahedron, 2007, 63, 7285; J. J. van Gorp, J. A. J. M. Vekemans and E. W. Meijer, J. Am. Chem. Soc., 2002, 124, 14759.

6 M. Blomenhofer, S. Ganzleben, D. Hanft and H.-W. Schmidt, Macromolecules, 2005, 38, 3688; M. Kristiansen, P. Smith, H. Chanzy, C. Baerlocher, V. Gramlich, L. McCusker, T. Weber, P. Pattison, M. Blomenhofer and H.-W. Schmidt, Cryst. Growth Des., 2009, 9, 2556; F. Abraham, S. Ganzleben, D. Hanft, P. Smith and H.-W. Schmidt, Macromol. Chem. Phys., 2010, 211, 171; F. Abraham and H.-W. Schmidt, Polymer, 2010, 51, 913; M. Kristiansen, A. Greß, P. Smith, D. Hanft and H.-W. Schmidt, Polymer, 2006, 47, 249.

7 N. Mohmeyer, N. Behrendt, X. Zhang, P. Smith, V. Altstädt, G. M. Sessler and H.-W. Schmidt, Polymer, 2007, 48, 1612.

8 D. K. Kumar, D. A. Jose, P. Dastidar and A. Das, Chem. Mater., 2004, 16, 2332; N. Shi, H. Dong, G. Yin, Z. Xu and S. Li, Adv. Funct. Mater., 2007, 17, 1837; N. Shi, G. Yin, M. Han and Z. Xu, Colloids Surf., B, 2008, 66, 84.

9 For example see: S. M. Aharoni, Polym. Prepr. (Am. Chem. Soc., Div. Polym. Chem.), 1989, 30, 125; S. M. Aharoni and S. F. Edwards, Macromolecules, 1989, 22, 3361; M. E. Cosulich, S. Russo, S. Pasquale and A. Mariani, Polymer, 2000, 41, 4951.

10 X. Song, Y. Zou, X. Liu, M. Oha and M. S. Lah, New J. Chem., 2010, 34, 2396; R. Ma, C. Chen, B. Sun, X. Zhao and N. Zhang, Inorg. Chem. Commun., 2011, 14, 1532.

11 S. Y. Ryu, S. Kim, J. Seo, Y.-W. Kim, O.-H. Kwon, D.-J. Jang and S. Y. Park, Chem. Commun., 2004, 1, 70.

12 D. J. Adams, M. F. Butler, W. J. Frith, M. Kirkland, M. Mullen and P. Sanderson, Soft Matter, 2009, 5, 1856; L. Chen, K. Morris, A. Laybourn, D. Elias, M. R. Hicks, A. Rodger, L. Serpell and D. J. Adams, Langmuir, 2010, 26, 5232.

13 Although the use of mineral acids (i.e. concentrated aqueous $\mathrm{HCl}$ ) instead of GdL results in very fast hydrogel formation and a much lower final $\mathrm{pH}$ value, the PL spectra of the resulting hydrogel samples are comparable to those obtained with the GdL method.

14 F. D. Lewis, T. M. Long, C. L. Stern and W. Liu, J. Phys. Chem. A, 2003, 107, 3254; A. Rochefort, E. Bayard and S. Hadj-Messaoud, Adv. Mater., 2007, 19, 1992.

15 The B3LYP functional was used in combination with the dispersion correction of Grimme, in order to take into account $\pi-\pi$ interactions: S. Grimme, J. Comput. Chem., 2004, 25, 1463.

16 J. Luo, Z. Xie, J. W. Y. Lam, L. Cheng, H. Chen, C. Qiu, H. S. Kwok, X. Zhan, Y. Liu, D. Zhu and B. Z. Tang, Chem. Commun., 2001, 1740; R. Deans, J. Kim, M. R. Machacek and T. M. Swager, J. Am. Chem. Soc., 2000, 122, 8565; M. K. Nayak, B.-H. Kim, J. E. Kwon, S. Park, J. Seo, J. W. Chung and S. Y. Park, Chem.-Eur. J., 2010, 16, 7437; Y. Hong, J. W. Y. Lam and B. Z. Tang, Chem. Soc. Rev., 2011, 40, 5361 .

17 Comparable conclusions were drawn regarding computational studies on other hydrogen-bonded supramolecular aggregates: A. Demenev, S. H. Eichhorn, T. Taerum, D. F. Perepichka, S. Patwardhan, F. C. Grozema, L. D. A. Siebbeles and R. Klenkler, Chem. Mater, 2010, 22, 1420.

18 The observed large Stokes shift may also be caused by some kind of orientational disorder inside the column (the emission then coming from defects after energy transfer along a supramolecular fiber), as shown in a theoretical work of Markovitsi and co-workers. The energy transfer mechanism of this class of compound is currently under investigation and will be subject of a full paper. See also: D. Markovitsi, Mol. Cryst. Liq. Cryst., 2003, 397, 89; D. Markovitsi, S. Marguet, J. Bondkowski and S. Kumar, J. Phys. Chem. B, 2001, 105, 1299; D. Markovitsi, A. Germain, P. Millié, P. Lécuyer, L. K. Gallos, P. Argyrakis, H. Bengs and H. Ringsdorf, J. Phys. Chem., 1995, 99, 1005.

19 C. F. C. Fitié, W. S. C. Roelofs, M. Kemerink and R. P. Sijbesma, J. Am. Chem. Soc., 2010, 132, 6892; A. Sakamoto, D. Ogata, T. Shikata, O. Urakawa and K. Hanabusa, Polymer, 2006, 47, 956. 